\title{
Improving the Understanding of Catalytic Processes using Field Emission Techniques: The Case of $\mathrm{NO}+\mathrm{H}_{2}$ on Pd-Au.
}

\author{
Cédric Barroo ${ }^{1,2}$ and Thierry Visart de Bocarmé ${ }^{1,2}$ \\ ${ }^{1}$ Chemical Physics of Materials and Catalysis, Université libre de Bruxelles, CP243, 1050 Brussels, \\ Belgium \\ ${ }^{2}$ Interdisciplinary Center for Nonlinear Phenomena and Complex Systems (CENOLI), Université libre \\ de Bruxelles (ULB), 1050 Brussels, Belgium
}

Catalysis plays a vital role in modern industrial applications. In the frame of automotive pollution control, dispersed nanoparticles are commonly used to reduce the emission of toxic gas species. To improve the efficiency of such processes, fundamental studies of the catalytic process is required at the single nanoparticle level in order to understand how the structure and composition of the catalytic material influence the reaction, and reversely, how the catalytic reaction influences the structure and composition of the catalyst. Indeed, local morphological and compositional changes occurring during the reaction might dramatically affect the reactivity and/or selectivity of the catalyst. To study these effects, we make use of the high-resolution capabilities of field emission techniques. Samples are prepared in the form of sharp tips and are used as model catalysts since the size $(\approx 20 \mathrm{~nm})$ and shape of the extremity of the sample mimics the structure of a single nanoparticle. In this work, Pd-38at\% Au [1] alloys are used to study the synergistic effects in the alloy as compared to isolated metals during the hydrogenation of NO.

In this context, we used field ion microscopy (FIM) to determine the structure of the catalyst before reaction, the morphological changes occurring during the adsorption of reactive gases, as well as the dynamics of reaction. The composition of the adsorbate layer was then probed by one-dimensional atom probe (1DAP). Finally, the atom probe tomography (APT) was used to investigate the surface composition of the sample before and after adsorption to highlight the presence of surface segregation. Samples are prepared as sharp tips by electrochemically etching a Pd-Au wire in a 10-20\% aqueous solution of $\mathrm{KCN}$ (with a potential of $3 \mathrm{~V}_{\mathrm{DC}}$ ). After introduction in the microscope, samples are cleaned by in situ treatments: cycles of thermal annealing, field evaporation and $\mathrm{Ne}^{+}$-ion sputtering.

The sample is first characterized by FIM at low temperature to analyze the crystallographic structure of the sample. Even though the presence of two different metals with two different lattice parameters may induce an imperfect FIM image (as compared to pure metals), it is possible to observe different crystallographic orientations with very good accuracy (Figure 1.a). Under FIM imaging, exposing this surface to NO gas at $400 \mathrm{~K}$ induces a morphological reconstruction from a quasi-hemispherical to a polyhedral shape. The equilibrium structure shows extended dense facets, such as $\{113\}$, to the detriment of more open facets such as $\{012\}$. To understand this, 1DAP is performed during the adsorption process. By using the combination of a static field and a pulsed field, it is possible to desorb the adsorbate layer or the atoms of the surface if the amplitude of the pulses is high enough. As presented Figure 1.b, the mass spectra mainly contain $\mathrm{NO}^{+}, \mathrm{N}^{+}, \mathrm{O}^{+}$and $\mathrm{NO}_{2}{ }^{+}$species, which prove the occurrence of NO dissociation in these experimental conditions. Due to the dissociation of the NO gas species, the presence of adsorbate and subsurface oxygen atoms is possible and might be responsible for the observed reconstruction. It must be mentioned that experiments performed on pure Pd [2] and pure $\mathrm{Au}$ [3] showed the presence of $\mathrm{NO}_{2}{ }^{+}$and $(\mathrm{NO})_{2}{ }^{+}$, and indicates that a disproportionation process must be 
considered in the mechanism of NO dissociation. The presence of both $\mathrm{Pd}$ and $\mathrm{Au}$ in the system allows the dissociation of $\mathrm{NO}$ to occur and could be due by the synergy between Au and Pd atoms. The next step towards the understanding of the system is to characterize the composition of the catalytic material before and after adsorption. For this, APT experiments are performed with similar tip-samples. The system is equipped with a reaction-cell allowing to expose the sample at temperatures up to $800-900 \mathrm{~K}$ under pressures up to $1 \mathrm{~atm}$ without external contaminations inherent to the transfer between microscopes. A reconstruction of the very first layer of the sample before any treatment is represented Figure 1.c and corresponds to the initial concentration of Pd-38at\% Au. After exposure to NO at $573 \mathrm{~K}$, the concentration of the surface evolves to $\mathrm{Pd}-20 \mathrm{at} \% \mathrm{Au}$, sign of Pd enrichment at the surface. Finally, the $\mathrm{NO}+\mathrm{H}_{2}$ reaction can be imaged during the ongoing process in FIM mode.

As a conclusion, the combination of different field emission techniques, i.e. field ion microscopy (FIM), one-dimensional atom probe (1DAP) and atom probe tomography (APT), allows to get a relatively complete picture of the catalytic system, from both the catalytic material and the catalytic reaction points of view. These techniques are particularly suitable to study the behavior of catalytic systems, with nanoscale resolution and at the single nanoparticle level. This is relevant to study the synergy between different metals in alloys which are increasingly used in industrial applications. [4]

\section{References:}

[1] C. Barroo, M. Moors and T. Visart de Bocarmé. Catal. Sci. Technol., 2017, 7, 5249-5256

[2] T. Visart de Bocarmé and N. Kruse, Ultramicroscopy, 2011, 111, 376-380

[3] T. D. Chau, T. Visart de Bocarmé and N. Kruse, Catal. Lett., 2004, 98, 85-87

[4] C.B. thanks the Fonds de la Recherche Scientifique (F.R.S.-FNRS) for financial support.

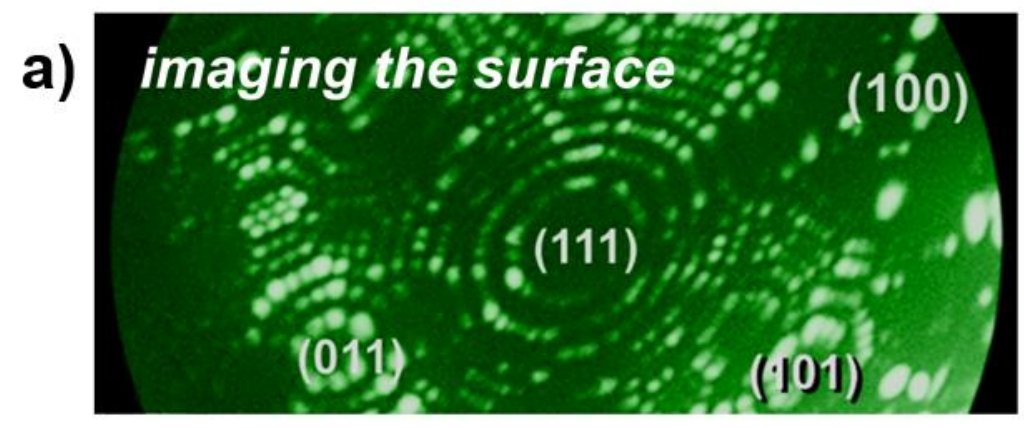

c) probing the volume

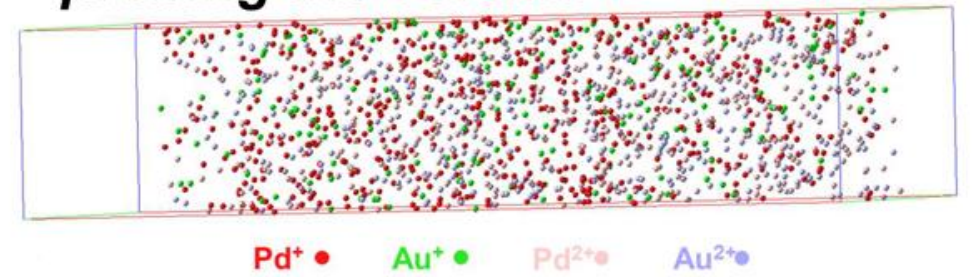

b) probing the surface

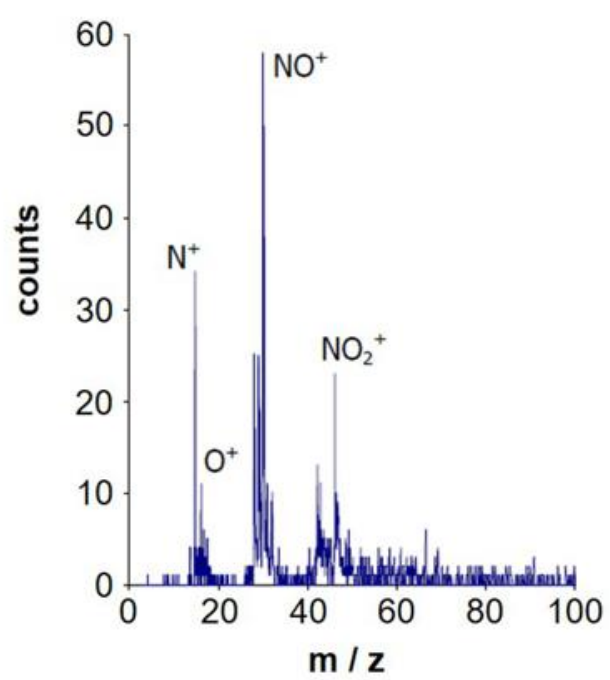

Figure 1. Characterization of Pd-38 at\%Au catalyst by field emission techniques: a) FIM imaging of a (111)-oriented tip before reaction; b) 1DAP analysis during the $\mathrm{NO}$ adsorption at $300 \mathrm{~K}$. The presence of $\mathrm{N}^{+}, \mathrm{O}^{+}$and $\mathrm{NO}_{2}{ }^{+}$is a strong indication of $\mathrm{NO}$ dissociation; $\left.\mathbf{c}\right) 3 \mathrm{D}$ reconstruction of the sample before any reaction showing a homogeneous distribution of $\mathrm{Pd}$ and $\mathrm{Au}$ atoms within the probed region corresponding to $\sim 10$ atomic layers (Image reproduced from [1]). 